\title{
Böttcher, Judith Marie (2017): Wie wirkt Planung? Theorie und Praxis der strategischen Stadtentwicklungsplanung am Beispiel Wohnen in wachsenden Großstädten.
}

\author{
Lemgo: Verlag Dorothea Rohn. 325 S.
}

\section{Gisela Schmitt ${ }^{1}$}

Eingegangen: 4. Februar 2018 / Angenommen: 12. April 2018 / Online publiziert: 16. April 2018

(c) Springer-Verlag GmbH Deutschland, ein Teil von Springer Nature 2018

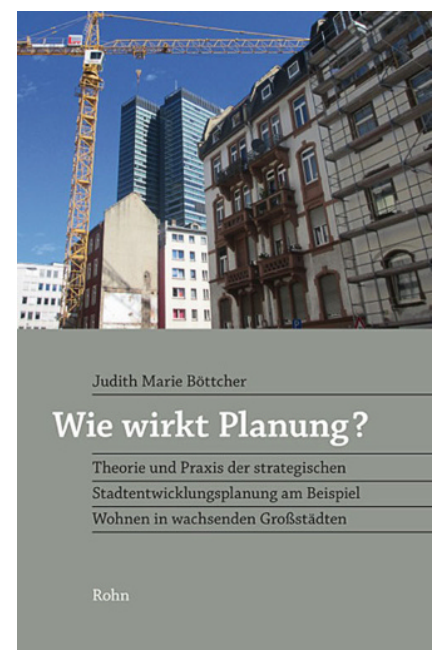

Wie wirkt Planung? Diese einfache Frage in der Überschrift erzeugt sicherlich hohe Aufmerksamkeit und Neugier bei all denen, die sich in Theorie und Praxis mit dem Planen beschäftigen. Aber auf einfache Fragen folgen nicht per se einfache Antworten - so auch nicht in dem vorliegenden Buch von Judith Marie Böttcher. Die Autorin befasst sich mit der genannten Frage in einer umfassenden und komplexen Abhandlung, die aus ihrer Dissertation hervorgegangen ist. Sie richtet den inhaltlichen Fokus auf Theorie und Praxis der strategischen Stadtentwicklung und wählt für ihre Fallstudien im Handlungsfeld Wohnen die Städte Frankfurt am Main und Münster aus.

Gisela Schmitt

schmitt@pt.rwth-aachen.de

1 Lehrstuhl für Planungstheorie und Stadtentwicklung, Rheinisch-Westfälische Technische Hochschule Aachen, Wüllnerstraße 5b, 52056 Aachen, Deutschland
Bei der theoretischen Einbettung der Forschungsfragen konzentriert sich die Autorin auf die strategische Stadtentwicklungsplanung. Planungswissenschaftliche Dissertationen können in der Regel auf kein konsistentes Theoriegerüst zurückgreifen und so steht auch Judith Marie Böttcher vor der Herausforderung, ihren gewählten Forschungsansatz im Theorienpluralismus der unterschiedlichen disziplinären Zugänge zu verorten. Neben der planungstheoretischen Literatur bezieht die Autorin auch ausgewählte Forschungsansätze der Management- und Organisationsforschung sowie der Politik- und Sozialwissenschaften ein. Strategien in der räumlichen Planung und im Management ordnet sie rückblickend bis zu den 1960er-Jahren in die verschiedenen Planungsverständnisse und Steuerungsansätze ein. Daneben greift sie die Governance-Perspektive als geeigneten Analyserahmen für strategische Stadtentwicklungsplanung auf, ebenso wie die Erkenntnisse aus dem Bereich des unternehmerischen Managements und der kommunalen Entscheidungsprozesse. Im Großen und Ganzen gelingt es der Autorin in der Auseinandersetzung mit der umfassenden Literatur und den unterschiedlichen wissenschaftlichen Positionen, in einer synoptischen Gegenüberstellung der Ansätze den notwendigen theoretischen Rahmen ihres Forschungsansatzes abzustecken und ein Gerüst von Leitfragen für ihre empirische Untersuchung abzuleiten. Im Fokus ihres Ansatzes steht die Strategiebildung in der Stadt- und Regionalentwicklung, die sie analytisch auf das (normative) Prozessmodell von Wiechmann (2008) stützt.

Judith Marie Böttcher interessiert nach ihrer eigenen Aussage an der strategischen Stadtentwicklungsplanung in erster Linie die Anwendung, Umsetzung und Wirkung in der Praxis. Daher erscheint es nur folgerichtig, dass sie ihre empirische Untersuchung als Evaluation konzipiert. Sie entwickelt auf der Grundlage der einschlägigen Literatur zur 
Wirkungsanalyse und der theoretischen Erörterungen zum Forschungsgegenstand ein eigenständiges Evaluationskonzept, dessen Untersuchungsdesign sie in einem Kapitel zur Methodik herleitet und erläutert. So bietet sich dem interessierten Leser die Gelegenheit, sich ,by the way“ auch noch auf den Stand der Debatte zur Wirkungsanalyse in der Stadtentwicklung zu bringen. Dass Evaluationen in der Stadtentwicklung eine besondere Herausforderung darstellen, ist spätestens seit den umfassenden Veröffentlichungen von Gerd-Michael Hellstern und Hellmut Wollmann in den 1980er-Jahren bekannt (vgl. z. B. Hellstern/Wollmann 1984), die Autorin geht hierauf in ihren Ausführungen auch ein (S. 81 ff.). Aber man hätte sich gewünscht, dass ihre Erkenntnisse zu Möglichkeiten und Grenzen der Wirkungsanalyse, die sie im Schlusskapitel reflektiert, schon vorab Eingang in das von ihr entwickelte Evaluierungskonzept gefunden hätten.

Den Fallstudien geht ein allgemeines Kapitel zum „Wohnen in wachsenden Großstädten“ voraus, welches die aktuellen Herausforderungen, vor denen die lokale Ebene heute in der Praxis steht, aufzeigt, um sehr systematisch den Handlungsspielraum der Städte anhand des Instrumentariums und der handelnden Akteure zu erläutern. An der ansonsten sehr sorgfältigen Ableitung irritiert ein wenig, dass das Verhältnis von Stadtentwicklungsplanung und Wohnungspolitik nicht geklärt wird. Ob sich kommunale Konzepte zum Wohnen, die komplexe ineinander verwobene Handlungssysteme abbilden, als Beispiele für strategische Stadtentwicklungskonzepte eignen - diese Frage wirft die Autorin nicht explizit auf. Ferner verzichtet sie darauf, das Handlungsfeld Wohnen aus einer theoretischen Perspektive und damit auch die Besonderheiten des Wohnungsmarktes näher zu beleuchten. Dies wäre für die Konzeption und spätere Auswertung ihrer Fallstudien hilfreich gewesen.

Angesichts akuter Problemlagen und zunehmendem Handlungsdruck sind seit Mitte der 2000er-Jahre vielerorts in den Städten der Wachstumsregionen übergreifende - oft auch strategisch ausgerichtete - Handlungskonzepte zum Wohnen entstanden, die Anlässe bieten, in der Auseinandersetzung zwischen Theorie und Praxis näher untersucht zu werden. „Wohnen in der Stadt“ stellt allerdings kein kohärentes planerisches Handlungsfeld, sondern ein kompliziertes lokales Handlungssystem zwischen Politik und Planung dar, das vielfach abhängig ist von den ordnungsund förderpolitischen Entscheidungen auf den übergeordneten Ebenen von Bund und Land. Die Tatsache, dass das Wohnen in unserem gesellschaftlichen System gleichermaBen ein Sozialgut wie auch Wirtschaftsgut darstellt, zwingt sowohl Politik als auch Planung auf der lokalen Ebene immer wieder in oft widersprüchliche Handlungsweisen, die eine Spannbreite von der Daseinsvorsorge (sozialverantwortliche Wohnungsversorgung) bis zur Wirtschaftsförderung (strategische Wohnstandortentwicklung) umfassen.
Unter Bezugnahme auf Schubert (2012: 29) stellen insbesondere die Entwicklungen in den Jahren zwischen 2004 und 2014, welche die Autorin in das Zentrum ihrer Betrachtung stellt, „das gesamte System der Wohnungsbauund Wohnungsmarktpolitik [...] auf den Prüfstand“ (S. 42). Vor diesem Hintergrund erscheint es zwar verständlich, den Bedarf nach einer ,umfassenden Wirkungsanalyse“ zu konstatieren (S. 42f.), aber angesichts der Überkomplexität des Gegenstandes auch von vorneherein nicht sehr zielführend, dies flächendeckend für kommunale Konzepte zum Wohnen zu versuchen.

Die beiden Fallstudien geben einen faktenreichen Überblick über die Strategien und Instrumente im Handlungsfeld Wohnen, der sowohl in die Chronologie der (strategischen) Stadtentwicklung als auch in allgemeine und wirtschaftliche Rahmenbedingungen eingebunden wird. Vor allem die Akteure, ihre Handlungsorientierung und Interaktionen werden durch Auswertung zahlreicher Interviews in die Betrachtung einbezogen. Die Ergebnisse zeigen unter anderem den starken Einfluss der veränderten Rahmenbedingungen und die Bedeutung der ,Windows of Opportunities“ auf die Wirkung der strategischen Konzepte (S. 258). Das Nebeneinander von rationalen, strategisch ausgerichteten Prozesselementen und kurzfristig orientierten inkrementalistischen Handlungsweisen zentraler Akteure, der eingesetzte Instrumentenmix und nicht zuletzt die kommunale Haushaltssituation bestimmen die unzureichende Umsetzung der Strategien. In dem differenziert gezeichneten Bild der zahlreichen, nicht widerspruchsfreien Handlungsansätze zum Wohnen auf der lokalen Ebene - im dem empirischen Fundus zu den realen Entwicklungen in zwei Großstädten über einen Zeitraum von zehn Jahren - liegt dann auch ein großer Verdienst der Studie.

Die Synthese zum Schluss gestaltet sich schwierig; es gelingt der Autorin nur bedingt, die Erkenntnisse über die komplexen realen Prozesse im Handlungsfeld Wohnen in differenzierte, empirisch begründete Ableitungen zur Strategiebildung in der Stadtentwicklung zu übertragen und mit der eingangs geführten breiten Theoriediskussion zu verschränken. Gleichwohl sind die empirischen Befunde zu Prozessen, Akteuren, Interaktionen und Konstellationen im Detail für einzelne Ansätze der Planungstheorie oder der Strategieforschung spannend. Es bleibt anzuerkennen, dass die Autorin die Erfahrungen und Ergebnisse aus ihrem Forschungsvorhaben in den Schlusskapiteln selbstkritisch und sehr differenziert reflektiert - so zum Beispiel die Weiterentwicklung der Evaluationsmethodik. Ihre abschließenden Überlegungen zur besseren Verschränkung von Theorie und Praxis im Feld der strategischen Stadtentwicklungsplanung können wertvolle Hinweise für nachfolgende Forschungen und Debatten liefern.

Die Autorin schreckt vor ,Komplexität' nicht zurück: Sie betreibt einen hohen Aufwand, um die Wissensgrund- 
lagen aus dem disziplinären Theorienpluralismus abzuleiten, wählt einen Forschungsgegenstand, der sich einer eindeutigen Einordnung weitgehend entzieht und stellt sich den Herausforderungen einer umfassenden Evaluation in zwei Fallstudien. Ihre Vorgehensweise bedarf meist umfassender Ausführungen zur Verortung, eröffnet jedoch auch neue vielversprechende Perspektiven - unter anderem zur empirischen Fundierung von Planungstheorien. Die Struktur der Dissertation prägt die Dramaturgie des vorliegenden Buches, das inhaltlich mit der Planung und dem Wohnen ein weites Feld aufspannt und - somit in Gänze gelesen dem fachlich interessierten Leser Durchhaltevermögen ab- verlangt. Gleichzeitig erlauben der systematische Aufbau des Buches und die stringente Abhandlung der einzelnen Kapitel auch erkenntnisreiche thematische Quereinstiege.

\section{Literatur}

Hellstern, G.-M.; Wollmann, H. (Hrsg.) (1984): Handbuch zur Evaluierungsforschung. Band 1. Opladen.

Schubert, D. (2012): Wieder einmal: die „neue“ Wohnungsnot. Gleichzeitig Wohnungsmangel und Wohnungsleerstand. In: RaumPlanung 163, 4, 26-30.

Wiechmann, T. (2008): Planung und Adaption. Strategieentwicklung in Regionen, Organisationen und Netzwerken. Dortmund. 